\section{NFACOM: A new program for relating solutions in exploratory factor analysis}

\author{
URBANO LORENZO and PERE J. FERRANDO \\ Universidad "Rovira $i$ Virgili," Tarragona, Spain
}

A new version of the program FACOM (comparison of exploratory factor analysis solutions) is presented. The new version (NFACOM) greatly extends the possibilities of the previous versions. It is now possible to use congruence as well as least squares comparisons in both orthogonal and oblique situations. What is more, $n$ solutions, not just two, can be compared. Recently developed algorithms have been implemented; others, which have proved not to be theoretically well founded, have been omitted.

The program FACOM (Lorenzo \& Ferrando, 1996) was developed for comparing exploratory factor analysis (EFA) solutions in a variety of situations. Since its original presentation, FACOM has undergone considerable modifications which greatly extend its possibilities: Many recently developed procedures have been added, but others, which have proved not to be theoretically correct, have been removed. The purpose of this announcement is to describe the features and facilities of the new FACOM (NFACOM).

The new version of the program enables factors to be compared in the following situations, none of which were considered in the previous version:

1. Orthogonal comparison versus oblique comparison. The researcher should decide whether the factors in the final solutions are to be orthogonal or oblique. NFACOM can perform the comparison in both situations.

2. Procrustes comparison versus congruence comparison. The first method is based on a least squares rotation; the second is based on a proportional rotation. Because the first method is much more strict, it is recommended only when one of the solutions is a hypothetical one (i.e., a theoretical target matrix). When two solutions have been obtained from different samples, a congruence rotation is recommended (Brokken, 1983).

3. Two-solution comparison versus n-solution comparison. When only two solutions are to be compared, one of them is taken as a target and the other is rotated (Cliff, 1966). However, if more than two solutions are to be compared, all of them are jointly rotated to a common solution (ten Berge, 1977).

The new procedures implemented in NFACOM for the situations described, are shown in Figure 1. Note that oblique comparison has not been considered with $n$ solutions, because theoretical algorithms have yet to be developed.

Correspondence should be addressed to U. Lorenzo, Departamento de Psicologia, Universidad "Rovira i Virgili," carretera Valls s/n, 43007 Tarragona, Spain.
In addition to these new methods, the old CANONICAL and SCORECOMP procedures are still available in NFACOM (see Lorenzo \& Ferrando, 1996). However, according to ten Berge (1996), the method of Kaiser, Hunka, and Bianchini (1971) has a dubious theoretical basis, and has therefore been omitted in the new version.

The procedures shown in Figure 1 can now be briefly introduced.

\section{ORTHOPROC}

Input. Two orthogonal patterns (or the oblique patterns together with the correlation matrices between factors) for each solution.

Rotation. Procrustes orthogonal rotation (Cliff, 1966), in which the second solution is taken as a target.

Output. Coefficient of factorial congruence, discrepancy index, salient similarity index, and the Pearson $r$ between the corresponding factor loadings.

\section{ORTHOCON}

Input. Two orthogonal patterns (or the oblique patterns together with the correlation matrices between factors) for each solution.

Rotation. Congruence orthogonal rotation (Kiers \& Groenen, 1996), in which the second solution is taken as a target.

Output. Coefficient of factorial congruence, discrepancy index, salient similarity index, and the Pearson $r$ between the corresponding factor loadings.

\section{MULPROC}

Input. The $n$ orthogonal patterns (or the oblique patterns with the factor correlation matrices) for the $n$ solutions.

Rotation. Procrustes orthogonal rotation (ten Berge, 1977), in which all the solutions are rotated to a common solution and then to a common Varimax position (see ten Berge, 1984, for details).

Output. Coefficient of factorial congruence, discrepancy index, salient similarity index, and the Pearson $r$ between the factor loadings. All these indices are computed for each pair of solutions.

\section{MULCON}

Input. The $n$ orthogonal patterns (or the oblique patterns with the factor correlation matrices) for the $n$ solutions.

Rotation. Procrustes congruence rotation (Kiers \& Groenen, 1996), in which all the solutions are rotated to a common solution and then to a common Varimax position (see ten Berge, 1984, for details).

Output. Coefficient of factorial congruence, discrepancy index, salient similarity index, and the Pearson $r$ between the factor loadings. All these indices are computed for each pair of solutions. 


\begin{tabular}{|l|c|c|c|c|}
\cline { 2 - 5 } \multicolumn{1}{c|}{} & \multicolumn{2}{c|}{ Two-Solution } & \multicolumn{2}{c|}{ n-Solution } \\
\cline { 2 - 5 } \multicolumn{1}{c|}{} & Procrustes & Congruence & Procrustes & Congruence \\
\hline Orthogonal & ORTHOPROC & ORTHOCON & MULPROC & MULCON \\
\hline Oblique & OBLIPROC & OBLICON & none & none \\
\hline
\end{tabular}

Figure 1. The new procedures that allow factor comparison in the following situations: orthogonal versus oblique, procrustes versus congruence, and two-solutions versus $n$ solutions.

\section{OBLIPROC}

Input. Two oblique patterns with their corresponding factor correlation matrices.

Rotation. Procrustes oblique rotation (ten Berge \& Nevels, 1977), in which the second solution is taken as a target.

Output. Coefficient of factorial congruence, discrepancy index, salient similarity index, and the Pearson $r$ between the corresponding factor loadings.

\section{OBLICON}

Input. Two oblique patterns with their corresponding factor correlation matrices.

Rotation. Congruence oblique rotation (Mosier, 1939), in which the second solution is taken as a target.

Output. Coefficient of factorial congruence, discrepancy index, salient similarity index, and the Pearson $r$ between the corresponding factor loadings.

\section{A Brief Example}

All the procedures implemented in NFACOM can be computed with the use of program files written in ASCII. As an example, the reader may consider the following EXAMPLE.PRG program:

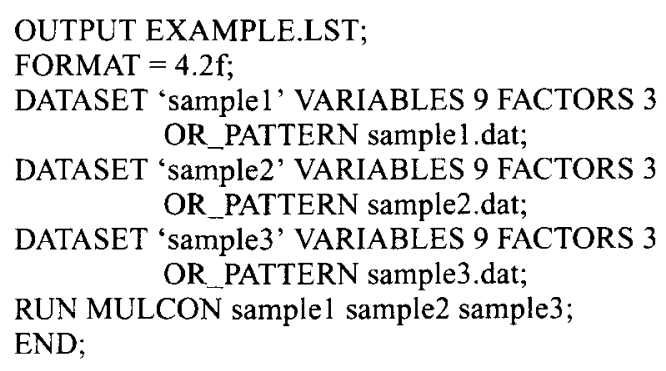

In this example, three orthogonal patterns have been stored in the files: sample1.dat, sample2.dat, and sample3.dat (in ASCII). All the patterns in the example have three factors and nine variables. With the MULCON procedure, they are all rotated to a common position and then to a Varimax position. To run this EXAMPLE.PRG file (in ASCII), the following DOS command is needed:

C:INFACOMINFACOM EXAMPLE.PRG
The OUTPUT file is EXAMPLE.LST (as defined in EXAMPLE.PRG).

\section{Software and Hardware Requirements}

NFACOM has been developed to run in a PC-compatible DOS environment. The computer should be a compatible 486 with at least $4 \mathrm{MB}$ of extended memory.

\section{Availability and Documentation}

NFACOM.EXE, some examples of application and a short manual can be obtained free of charge from the authors. Mail should be addressed to Urbano Lorenzo, Departamento de Psicologia, Universitat "Rovira i Virgili," Ctra de Valls s/n, 43007 Tarragona, Spain, or via electronic mail (uls@astor.urv.es). A diskette containing the program and the manual are available for $\$ 8$ U.S. to cover the cost of mailing. It can be also retrieved via anonymous ftp from trento.fcep.urv.es (IP 193.144.19.125)

\section{REFERENCES}

BRoKkEN, F. B. (1983). Orthogonal procrustes rotation maximizing congruence. Psychometrika, 48, 343-349.

ClifF, N. (1966). Orthogonal rotation to congruence. Psychometrika, 31, 33-42.

Kaiser, H. H., Hunka, S., \& Bianchini, J. C. (1971). Relating factors between studies based upon different individuals. Multivariate Behavioral Research, 6, 409-426.

KiERs, H. A. L., \& Groenen, P. (1996). A monotonically convergent algorithm for orthogonal congruence rotation. Psychometrika, 61, 375-389.

Lorenzo, U., \& Ferrando, P. J. (1996). FACOM: A library for relating solutions obtained in exploratory factor analysis. Behavior Research Methods, Instruments, \& Computers, 28, 627-630.

Mosier, C. I. (1939). Determining a simple structure when loadings for certain tests are known. Psychometrika, 4, 149-162.

TEN BERGE, J. M. F. (1977). Orthogonal procrustes rotation for two or more matrices. Psychometrika, 42, 267-276.

TEN BERGE, J. M. F. (1984). A joint treatment of varimax rotation and the problem of diagonalizing symmetric matrices simultaneously in the least-square sense. Psychometrika, 49, 347-358.

teN BERGE, J. M. F. (1996) The Kaiser, Hunka and Bianchini factor similarity coefficients: A cautionary note. Multivariate Behavioral Research, 31, 1-7.

TEN BERGE, J. M. F., \& NEvels, K. (1977). A general solution to Mosier's oblique procrustes problem. Psychometrika, 42, 593-600.

(Manuscript received April 21, 1997; revision accepted for publication September $30,1997$. ) 